\title{
Antidiabetic Micro-/Nanoaggregates from Ge-Gen-Qin-Lian-Tang Decoction Increase Absorption of Baicalin and Cellular Antioxidant Activity In Vitro
}

\author{
Dai Lin, ${ }^{1}$ Qian Du, ${ }^{2}$ Huiqin Wang, ${ }^{1}$ Guanzhen Gao, ${ }^{1}$ Jianwu Zhou, ${ }^{1}$ Lijing Ke, ${ }^{1}$ \\ Tianbao Chen, ${ }^{3}$ Chris Shaw, ${ }^{3}$ and Pingfan Rao ${ }^{1}$ \\ ${ }^{1}$ Food Nutrition Research Centre, School of Food Science and Biotechnology, Zhejiang Gongshang University, Hangzhou, \\ Zhejiang 310012, China \\ ${ }^{2}$ Institute of Biotechnology, Fuzhou University, Fuzhou, Fujian 350002, China \\ ${ }^{3}$ Natural Drug Discovery Group, School of Pharmacy, Queen's University Belfast, 97 Lisburn Road, Belfast, UK
}

Correspondence should be addressed to Lijing Ke; lijingke@zjgsu.edu.cn

Received 14 March 2017; Accepted 25 May 2017; Published 17 July 2017

Academic Editor: Sung-Hoon Kim

Copyright (C) 2017 Dai Lin et al. This is an open access article distributed under the Creative Commons Attribution License, which permits unrestricted use, distribution, and reproduction in any medium, provided the original work is properly cited.

\begin{abstract}
The antidiabetic effects of Ge-Gen-Qin-Lian-Tang decoction (GQD) have been proven clinically. In a pharmacological study conducted on STZ-induced diabetic rats, the constitutive aggregates/sediments of Ge-Gen-Qin-Lian-Tang decoction exhibited stronger hypoglycemic and antioxidant activities compared to the soluble compositions. This study aims to demonstrate the pharmacological properties of aggregates derived from GQD by measuring permeability of the active monomer phytochemicals (e.g., baicalin) in a Caco-2 cell monolayer and determine the cellular viability, intracellular redox status (MDA and SOD), and insulin secretion of pancreatic $\beta$-cell line, INS-1, following STZ-induced oxidative stress. The aggregates were separated into three fractions, namely, "MA (microaggregates)," " $400 \mathrm{~g}$ supernatant," and "MNA (micro-/nanoaggregates)," by centrifugation at $400 \times \mathrm{g}$ and $15000 \times \mathrm{g}$, respectively. Aggregates in the sediment increased baicalin absorption, showed little toxicity to $\beta$-cells, elevated intracellular SOD levels, and significantly suppressed oxidative damage effects on cellular viability and functions. The "MA" fraction had a larger particle size and provided higher antioxidant cellular protection than "MNA" in vitro, implying that the sediments may be the active components in the herbal decoction. The actions of these micro-/nanoaggregates may provide a new perspective for understanding the antidiabetic effects of herbal decoctions and aid in interpretation of synergistic actions between the multiple components.
\end{abstract}

\section{Introduction}

Herbal decoctions from traditional Chinese medicine (TCM) have always been an option for treating oxidative stressrelated chronic diseases [1-3], including diabetes. Many active components have been identified from various medicinal plants, for example, flavonoids [4], polyphenols [5], and organic acids and alkaloids, polypeptides, and polysaccharides $[1,6]$. These components may work alone as a single chemical compound or, in many instances, may deliver much more potent therapeutic effects in synergy with other components from the same plant or in a combination of different herbs, as demonstrated in many herbal TCM studies [7-9].
Besides the assumption that multiple components may be able to regulate several molecular pathways simultaneously, the rich content of colloidal particles and aggregates formed by various components in a decoction has been demonstrated in TCM (Ma-Xing-Shi-Gan-Tang). In one such instance, ephedrine and pseudoephedrine were mostly found to be bound to colloidal nanoparticles which changed their bioactivities [10] and this effect may provide the supramolecular structures for these synergistic actions.

As a classic herbal TCM dating back to the East Han Dynasty, Ge-Gen-Qin-Lian-Tang decoction (GQD) is prepared from a formula composed of Radix Puerariae Lobatae, Radix Scutellariae, Rhizoma Coptidis (Chinese goldthread), 
Radix Glycyrrhizae (licorice, honey-processed), and Zingiber officinale Roscoe (ginger) and has been used for the clinical treatments of type 2 diabetes and intestinal inflammation [11, 12]. GQD significantly reduced glycated hemoglobin (HbA1c) and fasting blood glucose (FBG) in streptozotocin- (STZ-) and high-fat-diet-induced diabetic SD rats and enhanced glucose consumption in 3T3-L1 adipocytes [13]. In T2D patients, GQD significantly reduced $\mathrm{HbAlc}$, while regulating the ecological structure of the gut microbiota by enriching the amounts of beneficial bacteria, indicating that the gut is among the important biological targets of this herbal tonic [12].

GQD contains several well-known active phytochemicals, that is, baicalin $[14,15]$, berberine $[16,17]$, puerarin $[18]$, glycyrrhizic acid, and liquiritin [19], which are correlated to the antidiabetic, antioxidant, and immunoregulative effects. Apart from its antihyperglycemic and antioxidant activities, baicalin is also known as a prolyl endopeptidase inhibitor which induces apoptosis in pancreatic cancer cells $[20,21]$ and it also induces apoptosis of lymphoma cells by downregulation of the PI3K/Akt signaling pathway [22]. However, taking the plant-derived flavone baicalin as an example, poor solubility and bioavailability are a common problem of these phytochemicals when applied clinically, resulting in the efficacy and pharmaceutical mechanisms of TCM decoctions being often questioned.

In our previous studies, GQD was found to exert antihyperglycemic effects on streptozocin- (STZ-) induced diabetic rats, while the micro-/nanoaggregates (sediments produced after high speed centrifugation) showed stronger activities than the supernatant on lowering blood glucose levels, elevating fasting blood insulin and insulin secretion index and increasing SOD activity of liver and pancreas [23]. These data implied that the micro-/nanoaggregates of TCM decoctions may account for their therapeutic effects, being at least as effective as the soluble components. The rich content of insoluble small molecules and self-assembled colloidal particles in these decoctions may account for the formation of micro-/ nanometer scale aggregates by secondary aggregation. The properties and functions of these aggregates warrant careful and systematic study.

To understand the cellular functions and antihyperglycemic mechanisms of aggregates in GQD, their particulate characteristics and effects on cell proliferation, insulin secretion, and redox status of pancreatic $\beta$-cells were assessed together with their impact on absorption of baicalin across Caco-2 colonic epithelial cell monolayers in vitro.

\section{Materials and Methods}

2.1. Materials. The herbs used in this study, for example, Radix Puerariae Lobatae, Radix Scutellariae, Rhizoma Coptidis (Chinese goldthread), Radix Glycyrrhizae (licorice, honey-processed), and sun-dried roots of Zingiber officinale Roscoe (ginger) were purchased from Beijing Yanjing Chinese Medicinal Herbs Co. Ltd. and authenticated by Professor Chengzi Yang from Fujian University of Traditional Chinese Medicine.
Cell culture medium (RPMI-1640, DMEM), HBSS buffer, penicillin-streptomycin solution, and NEAA were from HyClone (Xiamen, China); Fetal Bovine Serum (FBS) was from Biological Industries (BIOIND); HEHPE, 0.25\% Trypsin-EDTA, L-glutathione (reduced), and MTT were from AMRESCO Co. Ltd. (USA); streptozocin (STZ), sodium bicarbonate, and sodium pyruvate were from Sigma (USA). Baicalin was purchased from the Fujian Institute for Food and Drug Quality Control. Malondialdehyde (MDA), superoxide dismutase (SOD), and bicinchoninic acid (BCA) kits were from Nanjing Jiancheng Biotechnology Institute; ELISA kits for insulin measurements were purchased from Yanyu Biotech (Shanghai) Co. Ltd.

2.2. Preparation of GQD, $M A$, and $M N A$. Ge-Gen-Qin-LianTang decoction (GQD) was prepared by soaking Radix Puerariae Lobatae $72 \mathrm{~g}$, Radix Scutellariae $27 \mathrm{~g}$, Rhizoma Coptidis $27 \mathrm{~g}$, Radix Glycyrrhizae $18 \mathrm{~g}$, and ginger $4.5 \mathrm{~g}$ in $1.2 \mathrm{~L}$ deionized water for $30 \mathrm{~min}$ at $25^{\circ} \mathrm{C}$ and then boiled for $40 \mathrm{~min}$, cooled to room temperature, and filtered through two layers of cotton gauze. The filtrate was named GQD. After being centrifuged at $400 \times \mathrm{g}$ for $5 \mathrm{~min}$, the sediment of GQD was collected and resuspended with cell culture medium (the same volume as GQD) and named "microaggregates (MA)," while the supernatant was collected and named "400 g supernatant." Part of the supernatant was centrifuged at $15000 \times \mathrm{g}$ for $15 \mathrm{~min}$. The sediment was collected and resuspended to obtain the sample named "micro-/nanoaggregates (MNA)."

2.3. Determination of Particle Size. The hydrodynamic diameter of particles was determined by Dynamic Light Scattering (DLS) analysis on a Zetasizer Nano device (Malvern Instruments, Worcestershire, UK).

2.4. Cell Culture and MTT Assay. Human colonic epithelial cells, Caco- $2\left(8 \times 10^{4}\right.$ cells $/ \mathrm{mL}, 200 \mu \mathrm{L} /$ well $)$, and rat pancreatic $\beta$-cell line, INS-1 $\left(3 \times 10^{5}\right.$ cells $/ \mathrm{mL}, 200 \mu \mathrm{L} /$ well $)$, were cultured at $37^{\circ} \mathrm{C}$ under $5 \% \mathrm{CO}_{2}$ and $95 \%$ relative humidity for $24 \mathrm{~h}$ and used to evaluate the influence of GQD and aggregates on cellular viability and proliferation using the MTT assay. Samples were adjusted to the universal serial concentrations in terms of dry weight of herbs $(0.98,1.95$, $3.91,7.81,15.63,31.25,62.5$, and $125 \mathrm{mg} / \mathrm{mL}$ ), added to the cells in 96-well plates $(200 \mu \mathrm{L} /$ well), and cultured for $12 \mathrm{~h}$ with 5 replicates for each concentration. The test was repeated 3 times. The cell survival rate was calculated with the following equation (mean $\pm \mathrm{SD}, n=5$ ):

$$
\text { survival rate } \%=\frac{A_{570 \mathrm{~nm}} \text { sample }}{A_{570 \mathrm{~nm}} \text { control }} \times 100 \% .
$$

2.5. Permeability Tests on Caco-2 Cell Monolayers. Baicalin concentrations were determined by an RPLC method as previously reported [24]. An RPLC column, Daisogel-C18 (5 $\mu \mathrm{m}$, $4.6 \times 250 \mathrm{~mm})$, was used with methanol-water-phosphoric acid $(47: 53: 0.2)$ as eluting buffer, flow rate of $1.0 \mathrm{~mL} / \mathrm{min}$, monitored at $280 \mathrm{~nm}$; column temperature was set to $40^{\circ} \mathrm{C}$.

Permeability of GQD and its fractions was determined following a previously described protocol [25]. Briefly, the 
TABLE 1: Average diameter of aggregates in centrifuged sediments of GQD.

\begin{tabular}{lcc}
\hline Sample & Z-Average $d(\mathrm{~nm})$ & Derived count rate $(\mathrm{kcps})$ \\
\hline Microaggregates (MA) & $2775 \pm 712$ & $331 \pm 17$ \\
Micro-/nanoaggregates (MNA) & $531 \pm 23$ & $241 \pm 2$ \\
\hline
\end{tabular}

$n=3$; $Z$-Average $d(\mathrm{~nm})$ is the average diameter of particles. Derived count rate (kcps) partially indicates the particle concentration. MA: the resuspended sediment of GQD after low speed centrifugation $(400 \times \mathrm{g})$; MNA: the resuspended sediment of GQD obtained by high speed centrifugation $(15,000 \times \mathrm{g})$. Derived count rate: the intensity of light scattered by particles, presented as "thousand counts per second (kcps)."

cells were seeded onto Transwell plates and allowed to form a confluent monolayer over 20 days prior to the experiment. On day 21 , the test samples $(0.4 \mathrm{~mL})$, namely, GQD, MA, and MNA, were added to the apical side of the membrane and $0.6 \mathrm{~mL}$ HBSS buffer was added to the basolateral side. The transport of baicalin across the monolayer was monitored over a 3-hour time period at $37^{\circ} \mathrm{C}$ under $5 \% \mathrm{CO}_{2}$. Samples $(100 \mu \mathrm{L})$ were collected from the BL side at 30, 60, 90, 120, and 180 min. HBSS buffer $(100 \mu \mathrm{L})$ was added to the BL side each time the sample was collected. MA and MNA were dispersed evenly in cell culture medium by vortexing.

The permeability coefficient $\left(P_{\text {app }}\right)$ and absorption rate $(A \%)$ were calculated from the following equations $(n=3)$ :

$$
\begin{aligned}
P_{\mathrm{app}} & =\frac{(d Q / d t)}{A \times C_{0}} \\
A \% & =100 \times \frac{Q}{\left(C_{0} \times V\right)},
\end{aligned}
$$

where the unit of $P_{\text {app }}$ is $\mathrm{cm} / \mathrm{s}, d Q / d t$ is the rate of permeation of the drug across the cells $(\mu \mathrm{g} / \mathrm{s}), A$ is the area of cell monolayer, $C_{0}$ is the donor compartment concentration at time zero $(\mu \mathrm{g} / \mathrm{mL})$, and $Q$ is the total concentration of drug transfer across the cell monolayer $(\mu \mathrm{g}) . C_{0}$ is obtained from analysis of the dosing solution at the start of the experiment. $V$ is the volume of donor compartment.

\subsection{Effects of GQD, MA, and MNA on STZ-Induced Cellular} Damage. INS-1 cells were seeded into 96-well plates, grown for $24 \mathrm{~h}$ to form a confluent monolayer, and washed with PBS. GQD, MA, and MNA (each $100 \mu \mathrm{L}$ ) were added with $100 \mu \mathrm{L}$ STZ $\left(\mathrm{IC}_{50}\right)$ and incubated for $12 \mathrm{~h}$ prior to MTT assay. Five duplicates were used for each sample. The test was repeated 3 times. The cell survival rate was calculated with (1) and the protection rate was calculated with the following equation (means $\pm \mathrm{SD}, n=5$ ):

$$
\text { protection rate } \%=\frac{\left(A_{570 \text { sample }}-A_{570 \mathrm{STZ}}\right)}{\left(A_{570 \text { Normal }}-A_{570 \mathrm{STZ}}\right)} \times 100 \% \text {. }
$$

2.7. Effects of GQD, MA, and MNA on MDA, SOD, and Insulin Secretion. INS-1 cells $\left(5.5 \times 10^{6}\right.$ cells $)$ were seeded into 12 well plates and grown for $24 \mathrm{~h}$ to form a confluent monolayer. GQD, MA, and MNA (each $500 \mu \mathrm{L}$ ) were added with $500 \mu \mathrm{L}$ $\mathrm{STZ}\left(\mathrm{IC}_{50}=46.4 \mathrm{mM}\right)$ and incubated for $12 \mathrm{~h}$. Cells were then washed with $1 \mathrm{~mL}$ KRBB prior to the addition of $3.3 \mathrm{mM}$ glucose (dissolved in KRBB) and incubated for $1 \mathrm{~h}$. The culture supernatants $(500 \mu \mathrm{L})$ were collected and centrifuged at $4^{\circ} \mathrm{C}$ and $200 \times \mathrm{g}$ for $10 \mathrm{~min}$ and then stored at $-20^{\circ} \mathrm{C}$. The remaining $\mathrm{KRBB}$ was removed from cells before $16.7 \mathrm{mM}$ glucose (dissolved in KRBB) was gently added and incubated for $1 \mathrm{~h}$. The supernatant collection was then repeated as above. The cells were digested with pancreatin until $500 \mu \mathrm{L}$ of culture medium was added to stop the digestion. The cell suspension was moved to $1.5 \mathrm{~mL}$ tubes and centrifuged $\left(4^{\circ} \mathrm{C}, 200 \times \mathrm{g}\right.$, $10 \mathrm{~min}$ ) to collect cells for MDA, SOD, insulin (INS), and protein (bicinchoninic acid) assays.

2.8. Statistical Analysis. The raw data were processed with EXCEL (Microsoft, Inc.) and SigmaPlot (Systat Software, Inc.), and significance levels were determined by a one-way ANOVA and indicated as $P<0.05$ or $P<0.01$

\section{Results and Discussion}

3.1. Particle Size Distribution of GQD Aggregates. GQD was separated into three fractions by centrifugation. As shown in Figure 1, the aggregates in resuspended sediment produced after low speed centrifugation $(400 \times \mathrm{g})$ had an average diameter of $2 \sim 3 \mu \mathrm{m}$. The remaining particles in the supernatant were further separated with high speed centrifugation $(15,000 \times \mathrm{g})$ and resuspended to obtain a colloidal suspension with particles having an average diameter around $530 \mathrm{~nm}$ (Table 1) and a major size distribution from $300 \mathrm{~nm}$ to $1000 \mathrm{~nm}$. This centrifugation primarily separated the aggregates according to their average size and relative density, although some small colloidal particles may still remain in the supernatant of the $15,000 \times \mathrm{g}$ centrifugation.

3.2. Increased Baicalin Absorption. As demonstrated by Lin et al. [28], hydrophobic phytochemicals, that is, baicalin, puerarin, and berberine hydrochloride, are dispersed with the assistance of components from the constituent herbs, resulting in elevated solubility. In combined use with berberine, puerarin, glycyrrhizic acid, and liquiritin, the solubility and absorption of baicalin were improved $[29,30]$. As a purified component, baicalin is barely soluble in aqueous solution and has a very poor absorption rate of only $1 \%[26,27]$.

In this study, the intestinal absorption of baicalin from GQD was assessed in a Caco-2 cell monolayer model for evaluating whether formation of aggregates altered the bioavailability of Ge-Gen-Qin-Lian-Tang decoction (GQD). The baicalin-containing GQD showed 5-fold higher $P_{\text {app }}$ than baicalin alone (Table 2). MA contained $48 \%$ of decocting baicalin, exhibiting nearly twice $P_{\text {app }}$ and absorption rate in comparison to GQD. Meanwhile, MNA representing approx. $46 \%$ of total baicalin in the decoction exhibited 
TABLE 2: The apparent permeability $\left(P_{\text {app }}\right)$ and absorption rate of baicalin across Caco-2 cell monolayers.

\begin{tabular}{lcccc}
\hline Sample & Apical side baicalin concentration $(\mu \mathrm{g} / \mathrm{mL})$ & $P_{\text {app }}\left(\times 10^{-6} \mathrm{~cm} / \mathrm{s}\right)$ & Absorption rate in $3 \mathrm{~h}$ & Absorption level \\
\hline Pure baicalin ${ }^{*}$ & - & $0.66 \pm 0.10$ & $\sim 1 \%$ & Low \\
GQD & 27.6 & $3.40 \pm 0.21$ & $35 \%$ & Medium \\
MA & 12.9 & $6.60 \pm 0.18$ & $67 \%$ & High \\
400 g supernatant & 14.2 & $6.59 \pm 0.29$ & $66 \%$ & High \\
MNA & 12.8 & $7.30 \pm 0.17$ & $74 \%$ & High \\
\hline
\end{tabular}

${ }^{*}$ Data is cited from $[26,27] . n=4 . P_{\text {app }}>5 \times 10^{-6} \mathrm{~cm} / \mathrm{s}$ : high absorption; $P_{\text {app }}=1 \sim 5 \times 10^{-6} \mathrm{~cm} / \mathrm{s}$ : medium absorption; $P_{\text {app }}<1 \times 10^{-6} \mathrm{~nm} / \mathrm{s}:$ low absorption.

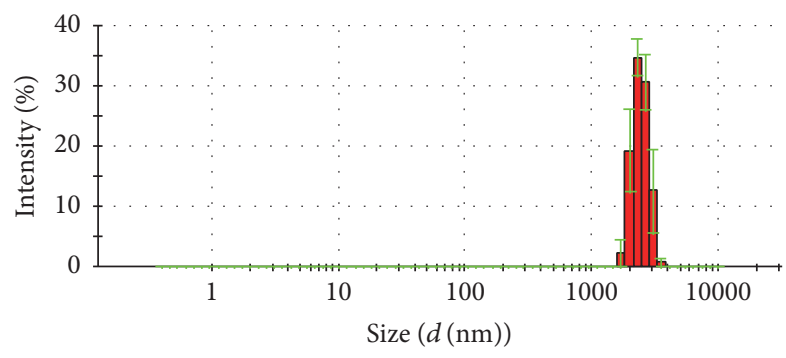

(a)

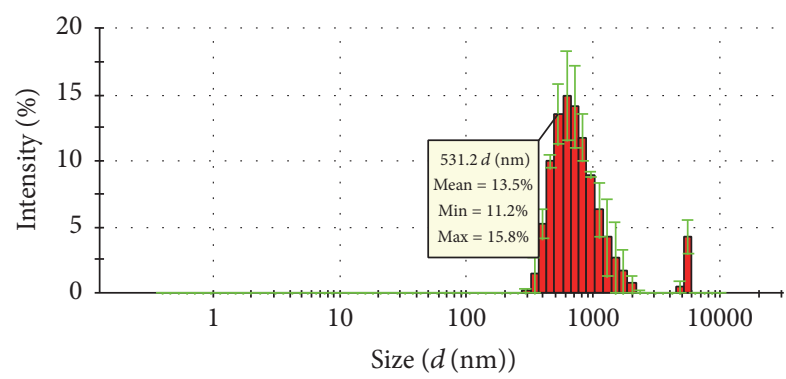

(b)

Figure 1: The particle size distribution of aggregates in GQD. (a) Particle size distribution of MA; (b) particle size distribution of MNA. Three duplicates were performed for each sample.

highest $P_{\text {app }}$ and absorption rate (Table 2). It is quite clear that the inclusion of baicalin in the sediments, even in terms of micrometer-scaled aggregates, strongly assisted transportation of Ge-Gen-Qin-Lian-Tang decoction (GQD) across the Caco- 2 cell monolayers, significantly increasing its absorption.

The absorption rate $(A \%)$ of baicalin in GQD aggregates across the Caco- 2 cells monolayer (from apical side to basolateral side) was determined at 30,60, 90, 120, and 180 min of incubation, as shown in Figure 2. Within the first $90 \mathrm{~min}$, the baicalin absorption rates of MA and MNA were the same. After incubation for a longer time ( $2 \mathrm{~h}$ and $3 \mathrm{~h}$ ), the MNA exhibited an $8 \%$ higher absorption than the MA, indicating that smaller particles may act as the more efficient vehicle for baicalin. Meanwhile, $A \%$ of baicalin in GQD were $19 \%$ at $30 \mathrm{~min}$ and $35 \%$ at $3 \mathrm{~h}$, which were lower than those of aggregates but higher than those of baicalin alone, implying a significantly improved absorption in the herbal suspension compared to the pure baicalin solution.

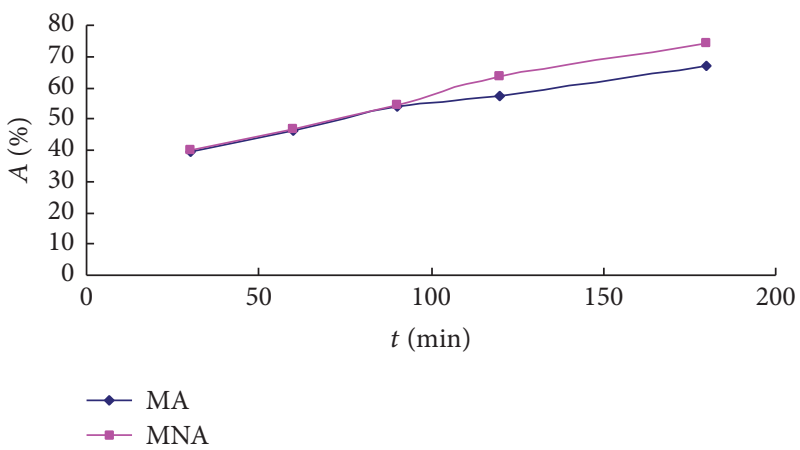

FIGURE 2: Absorption rate $(A \%)$ of baicalin in GQD aggregates on monolayers of Caco- 2 cells. Baicalin concentrations in the basolateral side solutions were determined by HPLC at different time points $(n=4)$.

It is well known that the glycyrrhizic acid (a licoricederived glycoside) is capable of forming intermolecular complexes to increase the solubility of poorly soluble drugs [31]. Our earlier work has also shown that even aqueous soluble plant-derived alkaloids (ephedrine) were mainly carried by colloidal nanoparticles self-assembled in another TCM herbal decoction and therefore exhibited different pharmacological characteristics from own monomer of GeGen-Qin-Lian-Tang decoction (GQD) [10]. As demonstrated above, higher $P_{\text {app }}$ and absorption rates of GQD aggregates indicate that the inclusion of baicalin in the higher order structures (i.e., supermolecular complexes and aggregates) changes pharmacokinetics of Ge-Gen-Qin-Lian-Tang decoction (GQD) and may be essential for its synergistic actions in the herbal decoction. Such complexes could be formed with flavonoids (such as puerarin and liquiritin), alkaloids (such as berberine), glycosides (such as baicalin and glycyrrhizic acid), polysaccharides, and glycated proteins.

\subsection{Influence of GQD and Its Aggregates on INS-1 Pancreatic} $\beta$-Cell Proliferation. As shown in Figure 3, GQD suppressed the growth of INS-1 pancreatic $\beta$-cells at $31.25 \sim 62.50 \mathrm{mg} / \mathrm{mL}$, implying a significant cytotoxicity $(P<0.01)$. However, at lower concentrations ( $15.63 \mathrm{mg} / \mathrm{mL}$ and lower), GQD showed no inhibition on the cell proliferation but rather promotion of such $(\max .60 \%$ at $7.81 \mathrm{mg} / \mathrm{mL}$ ). In contrast, the aggregates, both of MA and MNA, showed no cytotoxicity on INS-1 cells at concentrations as high as $125 \mathrm{mg} / \mathrm{mL}$. This indicates 

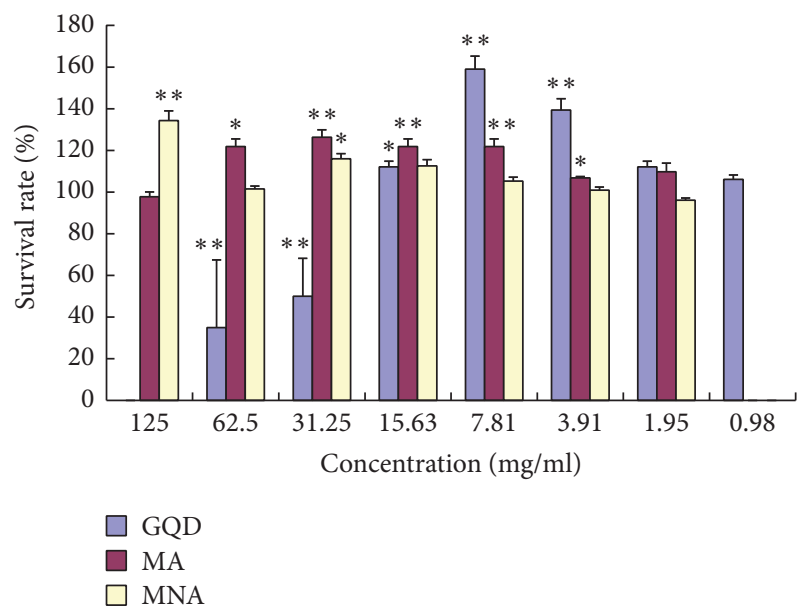

FIGURE 3: Effects of GQD and its aggregates on proliferation of INS-1 pancreatic $\beta$-cells. $n=5$. GQD (in blue): compared with normal controls, $0.98 \sim 1.95 \mathrm{mg} / \mathrm{mL}(P>0.05), 15.63 \mathrm{mg} / \mathrm{mL}(0.01<$ $P<0.05$, “*”), and others $(P<0.01$, “**”); MA (in orange): $400 \mathrm{~g}$ sediment, compared with normal controls, $125.0 \mathrm{mg} / \mathrm{mL}$ and $1.95 \mathrm{mg} / \mathrm{mL}(P>0.05), 62.50$ and $3.91 \mathrm{mg} / \mathrm{mL}(0.01<P<0.05$, “*”), and others $(P<0.01$, “**”); MNA (in grey): 15000 g sediment, compared with normal controls, $125.0 \mathrm{mg} / \mathrm{mL}(P<0.01$, “**”), $31.25 \mathrm{mg} / \mathrm{mL}(0.01<P<0.05$, “*”), and others $(P>0.05)$. Error bars + SEM. Differences are significant according to a oneway ANOVA indicated with an asterisk $(P<0.05 ; n=4)$ or double asterisks $(P<0.01 ; n=4)$.

that most of the cytotoxic compositions of GQD are in the supernatant after high speed centrifugation, which contains the majority of aqueous solutes. At medium concentrations (7.81 62.5 $\mathrm{mg} / \mathrm{mL}$ ), both MA and MNA mildly promoted cell proliferation, while the larger sized aggregates (MA) exhibited slightly higher proliferation rates, that is, $28 \%$ at $31.25 \mathrm{mg} / \mathrm{mL}$. The proliferation promoting activities of GQD and its aggregates may be attributed to their intracellular antioxidant capacities, since the pancreatic $\beta$-cells are sensitive to oxidative stress.

The effective concentration of GQD and its fractions appeared to be very high (in milligrams). It is because the concentration was presented in terms of the total dry weights of herbal materials used in preparing GQD. Given that the decocting only extracts a small portion of herbal materials, the dry weight of actual GQD dispersion and its aggregate fractions would be many times lower.

3.4. Inhibition of STZ-Induced Cellular Oxidation. As shown in Figure 4, GQD protected INS-1 cells from STZ-induced oxidative damage by $23 \%$ at $7.81 \mathrm{mg} / \mathrm{mL}$ but showed no protection at higher or lower concentrations. In comparison, both MA and MNA significantly protected the cells at a much wider range of concentrations (1.95 to $31.25 \mathrm{mg} / \mathrm{mL}$ ) and achieved much stronger protection (MA, 78\% at $7.81 \mathrm{mg} / \mathrm{mL}$ ). It indicates that antioxidants or components capable of elevating the cellular antioxidant capacity are embedded in the aggregates but not in the soluble fraction. Given that neither MA nor MNA exhibited cytotoxicity (Figure 3), the cytotoxic components of GQD are most likely to be in the

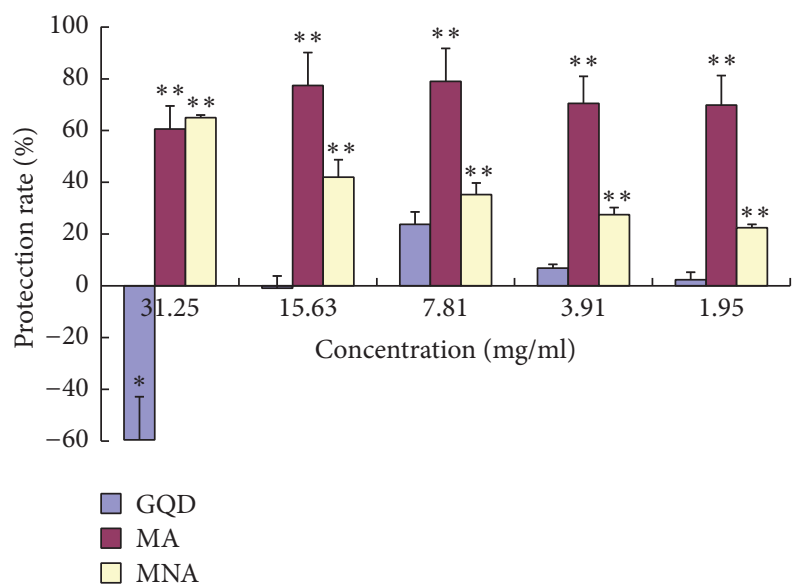

FIgURE 4: Protection of GQD and its aggregates against STZinduced oxidative suppression of the growth of INS-1 $\beta$-cells. $n=5$. Oxidative damage was induced with STZ at its $\mathrm{IC}_{50}(46.4 \mathrm{mM})$. GQD: compared with STZ controls, $31.25 \mathrm{mg} / \mathrm{mL}(0.01<P<$ 0.05 , labelled “*”) and others $(P>0.05)$; MA: $400 \mathrm{~g}$ sediment, compared with STZ controls, at all concentrations, $P<0.01$ (“**”); MNA: 15000 g sediment, compared with STZ controls, at all concentrations, $P<0.01$ (“**”).

supernatant. Meanwhile, the larger sized aggregates generally showed significantly higher protection rates $(P<0.01)$ than the smaller sized aggregates. Notably, the protection rates of MA were irrelevant to dosage, while those of MNA were dose-dependent, indicating that these two groups of aggregates may work via different mechanisms to inhibit cellular oxidative damage.

The significantly elevated MDA level (Table 3) and reduced SOD level (Table 4) indicated that INS-1 cells had been damaged by STZ-induced oxidation. The aggregates (both MA and MNA) significantly restored the cellular SOD activity and reduced the MDA level, whereas GQD only exhibited significant antioxidant effects at $7.81 \mathrm{mg} / \mathrm{mL}$. This is consistent with the different performance of the aggregates and GQD on regulating cellular viability (Figure 4). By comparing the MAD and SOD levels of GQD and its fractions, their antioxidant activities were ranked in sequence: $\mathrm{MA}>$ $400 \mathrm{~g}$ supernatant $>$ MNA $>$ GQD.

The supernatant is rather high in antioxidant activity but is toxic to the cells, implying that, at high GQD concentrations, the cytotoxic components overrule the antioxidant (cytoprotective) components and therefore kill the cells.

3.5. Restoration of Insulin Secretion. The impacts of STZinduced oxidation and GQD samples on insulin secretion from pancreatic $\beta$-cells were evaluated at either baseline levels $(3.3 \mathrm{mM})$ or stimulated levels $(16.7 \mathrm{mM})$ of glucose, as shown in Figure 5. The insulin secretion index (ISI) was calculated as a ratio of glucose-stimulated insulin secretion (GSIS)/basal insulin secretion (BIS) and data are shown in Figure 6.

STZ-induced oxidation reduced the expression and secretion of insulin, causing cells to be irresponsive to the glucose 
TABLE 3: GQD and components reduced STZ-induced MDA in pancreatic $\beta$-cell line, INS-1.

\begin{tabular}{|c|c|c|c|c|}
\hline \multirow{2}{*}{ Group } & \multicolumn{4}{|c|}{$\mathrm{MDA}(\mathrm{nmol} / \mathrm{mL})$} \\
\hline & GQD & MA & $400 \mathrm{~g}$ supernatant & MNA \\
\hline $7.81 \mathrm{mg} / \mathrm{mL}$ herbs & $9.40 \pm 0.47^{\mathrm{ab}}$ & $7.13 \pm 1.71^{\mathrm{ab}}$ & $8.29 \pm 1.07^{\mathrm{ab}}$ & $8.85 \pm 0.77^{\mathrm{ab}}$ \\
\hline $3.91 \mathrm{mg} / \mathrm{mL}$ herbs & $9.81 \pm 0.24^{\mathrm{ab}}$ & $7.41 \pm 1.55^{\mathrm{ab}}$ & $8.78 \pm 0.81^{\mathrm{ab}}$ & $9.17 \pm 0.60^{\mathrm{ab}}$ \\
\hline $1.95 \mathrm{mg} / \mathrm{mL}$ herbs & $10.06 \pm 0.12^{\mathrm{a}}$ & $7.49 \pm 1.51^{\mathrm{ab}}$ & $9.04 \pm 0.67^{\mathrm{ab}}$ & $9.37 \pm 0.50^{\mathrm{ab}}$ \\
\hline STZ control & \multicolumn{4}{|c|}{$10.25 \pm 0.24^{\mathrm{a}}$} \\
\hline Normal & \multicolumn{4}{|c|}{$5.89 \pm 0.17^{\mathrm{b}}$} \\
\hline
\end{tabular}

$n=5$; $^{\mathrm{a}}$ compared with normal cells $(P<0.05){ }^{\mathrm{b}}{ }^{\mathrm{b}}$ compared with STZ controls $(P<0.05)$.

TABLE 4: GQD and components restored SOD activity in STZ-treated pancreatic $\beta$-cell line, INS-1.

\begin{tabular}{|c|c|c|c|c|}
\hline \multirow{2}{*}{ Group } & \multicolumn{4}{|c|}{ SOD (U/mg.protein) } \\
\hline & GQD & MA & $400 \mathrm{~g}$ supernatant & MNA \\
\hline $7.81 \mathrm{mg} / \mathrm{mL}$ herbs & $83.95 \pm 5.32^{\mathrm{ab}}$ & $133.33 \pm 8.02^{\mathrm{b}}$ & $106.44 \pm 4.36^{\mathrm{ab}}$ & $93.77 \pm 8.87^{\mathrm{ab}}$ \\
\hline $3.91 \mathrm{mg} / \mathrm{mL}$ herbs & $66.22 \pm 7.60^{\mathrm{a}}$ & $127.40 \pm 8.40^{\mathrm{ab}}$ & $94.97 \pm 5.35^{\mathrm{ab}}$ & $85.63 \pm 3.69^{\mathrm{ab}}$ \\
\hline $1.95 \mathrm{mg} / \mathrm{mL}$ herbs & $62.00 \pm 6.56^{\mathrm{a}}$ & $125.33 \pm 5.51^{\mathrm{ab}}$ & $87.93 \pm 6.76^{\mathrm{ab}}$ & $80.60 \pm 4.23^{\mathrm{ab}}$ \\
\hline STZ Control & \multicolumn{4}{|c|}{$60.75 \pm 4.07^{\mathrm{a}}$} \\
\hline Normal & \multicolumn{4}{|c|}{$155.88 \pm 6.80^{\mathrm{b}}$} \\
\hline
\end{tabular}

$n=5$; $^{\text {a }}$ compared with normal cells $(P<0.05){ }^{\text {b }}$ compared with STZ controls $(P<0.05)$.

stimulus. The baseline insulin secretion of normal INS$1 \beta$-cells was $67 \mathrm{pg} / \mathrm{mL}$, which was dramatically increased threefold to the stimulated level of $214 \mathrm{pg} / \mathrm{mL}$ (ISI $=3.23$ ). In contrast, STZ-damaged cells did not respond to such a stimulus. The presence of GQD did not improve the baseline insulin secretion of STZ-damaged cells but doubled the insulin secretion ( $\max .97 \mathrm{pg} / \mathrm{mL}, P<0.01)$ at corresponding elevated glucose levels.

Aggregates from GQD, namely MA and MNA, significantly improved both the baseline and stimulated insulin secretions $(P<0.01$, Figure 5). Notably, the larger size aggregates (MA) showed much stronger restorative power than the MNA, wherein the BIS was almost fully restored and the GSIS (max. $174 \mathrm{pg} / \mathrm{mL}$, ISI = 2.7) was about twofold higher than that of MNA. The MNA significantly improved the GSIS ( $\max$. ISI $=1.9$ ) in a dose-dependent manner. Its overall effects were rather like GQD, except that the effective concentration of MNA was lower than that of GQD.

All the insulin secretion results were consistent with the cellular protection and antioxidant effects of GQD and its constitutive aggregates. The significant higher antioxidant activity of aggregates on cells was in good agreement with their antioxidant effects in vivo [23], wherein the aggregates elevated SOD levels in pancreas, kidney, and liver of STZinduced diabetic rats. Despite the higher bioavailability of baicalin in MNA demonstrated earlier in this study, the aggregates in MA showed more potent protective effects against STZ-induced oxidative stress upon cells. Although it remains unclear why larger size aggregation particles exhibited stronger antioxidant activity, one can anticipate that such aggregates may have a higher content of free radical scavenging compounds, such as berberine, puerarin, liquiritin, and glycyrrhizin acid [15-19, 32]. Besides, the MA is still a high absorption drug, whose absorption rate was only $7 \%$ lower than that of MNA. The slightly lower absorption rate could be compensated by the richer contents of particles in MA indicated by their higher scattering light intensity (kcps).

Although the higher absorption rate and antioxidant and cellular protective activities in vitro do not necessarily mean better therapeutic effectiveness in vivo, it is reasonable to anticipate that the micro-/nanoscale aggregates may have a vital contribution to the overall antidiabetic effects of the herbal decoction (GQD), noting that the herbal components would eventually interact with the mammalian digestive tract in the form of multiple-order aggregates, such as chyle.

\section{Conclusions}

The antidiabetic herbal tonic, GQD, contains micro- and nanoscale aggregates which improve the bioavailability of insoluble phytochemicals, that is, baicalin, and possess little cytotoxicity on colonic epithelial cells and pancreatic $\beta$ cells (INS-1) in vitro. It also elevates cellular antioxidant enzymes and protects $\beta$-cells from STZ-induced oxidation and restores their insulin secretion capability. The centrifugal separation results in two different size distribution fractions of aggregates (centrifuge sediments), and the larger size aggregates (MA) possessed stronger protection on cellular viability and function of $\beta$-cells in vitro. These data are consistent with an earlier antihyperglycemic study of GQD aggregates on STZ-induced diabetic rats. The aggregates from the TCM decoction, for the first time, have been found to contain active components that contribute to the antidiabetic activity of the herbal tonic by exhibiting antioxidant effects on the endocrine cells and the carrying of insoluble compounds across the intestinal mucosal barrier. These data also imply that the aggregates and sediments in the herbal decoction should be handled with greater care for both TCM herbal medicine production and pharmacological studies. 

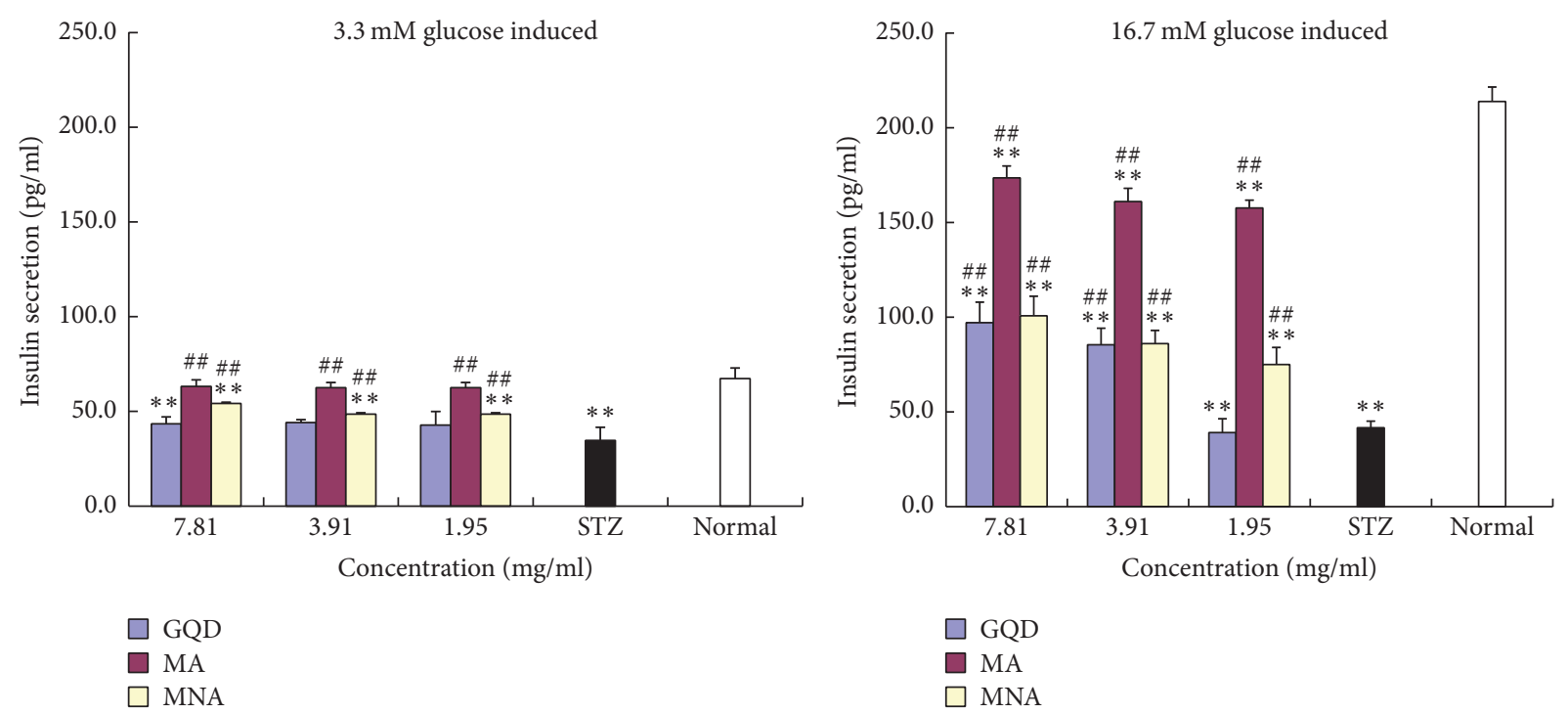

FIGURE 5: GQD and its aggregates restoration of insulin secretion in STZ-damaged INS-1 cells. “**”: compared with normal cells, $P<0.01$, $n=3$; “\#\#": compared with STZ controls, $P<0.01, n=3$.

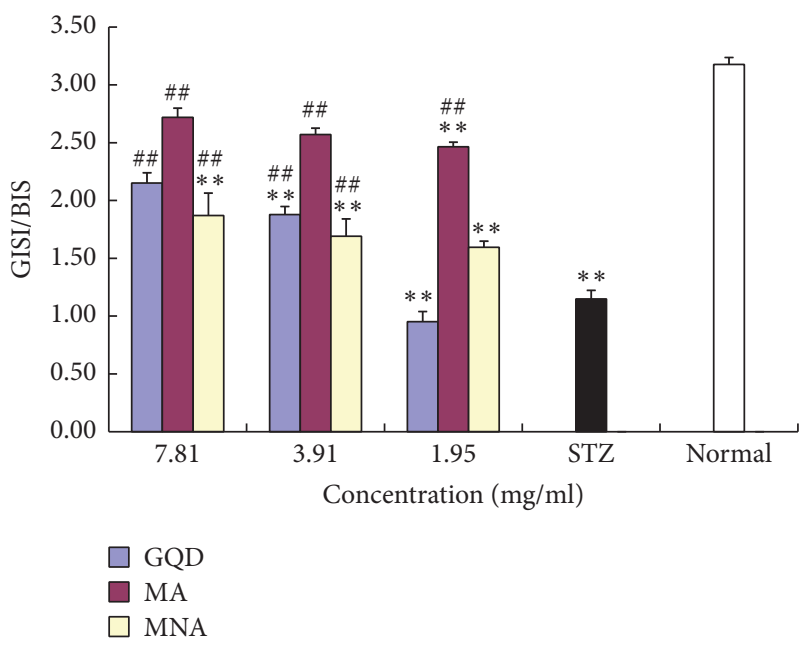

FIGURE 6: GQD and its constitutive aggregates elevation of insulin secretion index (ISI) of STZ-damaged INS-1 $\beta$-cells. “**”: compared with normal cells, $P<0.01, n=3$; “\#\#”: compared with STZ controls, $P<0.01, n=3$; ISI: GSIS/BIS.

In comparison with monomer compound studies, it would require different approaches to elucidate the pharmacological mechanisms underpinning the therapeutic actions of TCM aggregates and identify the constituent chemicals of aggregates in different size. The particulates can be further separated by ultrafiltration or size-exclusion chromatography or ion-exchange chromatography according to their physical size, for example, diameter, or surface charge. The phase extraction and/or enzymatic hydrolysis can then be applied to deconstruct these separated fractions of aggregates, whose chemical compositions will be resolved with chromatographic approach coupled with mass spectra. Thus, more comprehensive studies on these colloidal micro-/ nanoparticles and their constituent compounds are warranted to fully understand their pharmacological characteristics and chemical natures, which may inspire and lead to the development of active supramolecular complexes for the treatment of oxidative diseases.

\section{Conflicts of Interest}

All the authors declare that there are no financial conflicts of interest.

\section{Acknowledgments}

This research was supported by the National Key Research and Development Plan (2016YFD0400202), the National Natural Science Foundation of China (Grant no. 31571803), and the Zhejiang Provincial Natural Science Foundation of China (LY16C200001).

\section{References}

[1] Z. Wang, J. Wang, and P. Chan, "Treating type 2 diabetes mellitus with traditional chinese and indian medicinal herbs," Evidence-Based Complementary and Alternative Medicine, vol. 2013, Article ID 343594, 17 pages, 2013.

[2] Y. Zhong, Y. Deng, Y. Chen, P. Y. Chuang, and J. Cijiang He, "Therapeutic use of traditional Chinese herbal medications for chronic kidney diseases," Kidney International, vol. 84, no. 6, pp. 1108-1118, 2013.

[3] W. Xie, Y. Zhao, and Y. Zhang, "Traditional chinese medicines in treatment of patients with type 2 diabetes mellitus," EvidenceBased Complementary and Alternative Medicine, vol. 2011, Article ID 726723, 13 pages, 2011. 
[4] H. Liao, L. K. Banbury, and D. N. Leach, "Antioxidant activity of 45 Chinese herbs and the relationship with their TCM characteristics," Evidence-based Complementary and Alternative Medicine, vol. 5, no. 4, pp. 429-434, 2008.

[5] H. Liu, N. Qiu, H. Ding, and R. Yao, "Polyphenols contents and antioxidant capacity of 68 Chinese herbals suitable for medical or food uses," Food Research International, vol. 41, no. 4, pp. 363$370,2008$.

[6] T. Okuda, "Antioxidant Food Supplements in Human Health," 26-Antioxidants in Herbs: Polyphenols, pp. 393-410, 1999.

[7] M. A. Rather, B. A. Bhat, and M. A. Qurishi, "Multicomponent phytotherapeutic approach gaining momentum: Is the "one drug to fit all" model breaking down?" Phytomedicine, vol. 21, no. 1, pp. 1-14, 2013.

[8] F. Hong, W. Xiao, G. Ragupathi et al., "The known immunologically active components of Astragalus account for only a small proportion of the immunological adjuvant activity when combined with conjugate vaccines," Planta Medica, vol. 77, no. 8, pp. 817-824, 2011.

[9] K.-M. Lau, K.-K. Lai, C.-L. Liu et al., "Synergistic interaction between Astragali Radix and Rehmanniae Radix in a Chinese herbal formula to promote diabetic wound healing," Journal of Ethnopharmacology, vol. 141, no. 1, pp. 250-256, 2012.

[10] J. Zhou, G. Gao, Q. Chu, H. Wang, P. Rao, and L. Ke, "Chromatographic isolation of nanoparticles from Ma-XingShi-Gan-Tang decoction and their characterization," Journal of Ethnopharmacology, vol. 151, no. 3, pp. 1116-1123, 2014.

[11] X.-L. Tong, L.-H. Zhao, F.-M. Lian et al., "Clinical observations on the dose-effect relationship of gegen qin lian decoction on 54 out-patients with type 2 diabetes," Journal of Traditional Chinese Medicine, vol. 31, no. 1, pp. 56-59, 2011.

[12] J. Xu, F. Lian, L. Zhao et al., "Structural modulation of gut microbiota during alleviation of type 2 diabetes with a Chinese herbal formula," ISME Journal, vol. 9, no. 3, pp. 552-562, 2015.

[13] C.-H. Zhang, G.-L. Xu, Y.-H. Liu et al., "Anti-diabetic activities of Gegen Qinlian Decoction in high-fat diet combined with streptozotocin-induced diabetic rats and in 3T3-L1 adipocytes," Phytomedicine, vol. 20, no. 3-4, pp. 221-229, 2013.

[14] Y. Fu, J. Luo, Z. Jia et al., "Baicalein protects against type 2 diabetes via promoting islet $\beta$-cell function in Obese Diabetic Mice," International Journal of Endocrinology, vol. 2014, Article ID 846742, 2014.

[15] V. Y. Waisundara, S. Y. Siu, A. Hsu, D. Huang, and B. K. H. Tan, "Baicalin upregulates the genetic expression of antioxidant enzymes in Type-2 diabetic Goto-Kakizaki rats," Life Sciences, vol. 88, no. 23-24, pp. 1016-1025, 2011.

[16] Z. Li, Y. N. Geng, J. D. Jiang, and K.-J. Kong, "Antioxidant and anti-inflammatory activities of berberine in the treatment of diabetes mellitus," Evidence-Based Complementary and Alternative Medicine, vol. 2014, Article ID 289264, 12 pages, 2014.

[17] A. Luo and Y. Fan, "Antioxidant activities of berberine hydrochloride," Journal of Medicinal Plants Research, p. 3702, 2011.

[18] Y.-X. Zhou, H. Zhang, and C. Peng, "Puerarin: a review of pharmacological effects," Phytotherapy Research, vol. 28, no. 7, pp. 961-975, 2014.

[19] J. Cheel, P. V. Antwerpen, L. Tůmová et al., "Free radicalscavenging, antioxidant and immunostimulating effects of a licorice infusion (Glycyrrhiza glabra L.)," Food Chemistry, vol. 122, no. 3, pp. 508-517, 2010.
[20] T. Tarragó, N. Kichik, B. Claasen, R. Prades, M. Teixidó, and E. Giralt, "Baicalin, a prodrug able to reach the CNS, is a prolyl oligopeptidase inhibitor," Bioorganic and Medicinal Chemistry, vol. 16, no. 15, pp. 7516-7524, 2008.

[21] H. Takahashi, M. C. Chen, H. Pham et al., "Baicalein, a component of Scutellaria baicalensis, induces apoptosis by Mcl-1 down-regulation in human pancreatic cancer cells," Biochimica et Biophysica Acta - Molecular Cell Research, vol. 1813, no. 8, pp. 1465-1474, 2011.

[22] Y. Huang, J. Hu, J. Zheng et al., "Down-regulation of the $\mathrm{PI} 3 \mathrm{~K} / \mathrm{Akt}$ signaling pathway and induction of apoptosis in CA46 Burkitt lymphoma cells by baicalin," Journal of Experimental \& Clinical Cancer Research, p. 48, 2012.

[23] D. Wu, L. Ke, H. Liu et al., "Antidiabetic effects of Ge-Gen-QinLian-Tang decoction and its aggregated compositions on STZinduced diabetic Wistar rats," Journal of FuZhou university, vol. 42, pp. 1-6, 2014.

[24] D. Jing, W. Yan, and F. Ying-hua, "Determination of Baicalin in Huangqin Gargles by RP-HPLC," Chinese Arch Tradit Chinese Med, p. 30, 2012.

[25] X.-W. Yang, X.-D. Yang, Y. Wang et al., "Establishment of Caco2 cell monolayer model and standard operation procedure for assessing intestinal absorption of chemical components of traditional Chinese medicine," Journal of Chinese Integrative Medicine, vol. 5, no. 6, pp. 634-641, 2007.

[26] M.-L. Zhu, X.-L. Liang, L.-J. Zhao et al., "Elucidation of the transport mechanism of baicalin and the influence of a Radix Angelicae Dahuricae extract on the absorption of baicalin in a Caco-2 cell monolayer model," Journal of Ethnopharmacology, vol. 150, no. 2, pp. 553-559, 2013.

[27] Y. Wang, J. Jia, and X. Qin, "Comparison of the small intestinal absorption of baicalin and baicalin drop pills by Caco-2 cell model," Chinese J Drug Appl Monit, 2010.

[28] W. Lin, X. Long, W. Chen, and Y. Wang, "Solubility and oilwater partition coefficient of puerarin,baicalin and berberine hydrochloride in Gegenqinlian formula," J Guangdong Pharm Univ, 2014

[29] L. Chen, X.-Y. Long, S.-H. Huang, H.-Y. Wu, and S.-J. Pan, "[Drug delivery systems of baicalin, baicalin-phospholipid complex and self-microemulsifying drug across Caco-2 cell model]," Journal of Chinese Medicinal Materials, 2012.

[30] B. Xu, P. Li, and G. Zhang, "Comparative pharmacokinetics of puerarin, daidzin, baicalin, glycyrrhizic acid, liquiritin, berberine, palmatine and jateorhizine by liquid chromatographymass spectrometry after oral administration of Gegenqinlian decoction and active components alignment (ACA) to rats," Journal of Chromatography B: Analytical Technologies in the Biomedical and Life Sciences, vol. 988, pp. 33-44, 2015.

[31] V. Dushkin A, TG. Tolstikova, V. Khvostov M, and GA. Tolstikov, "Tolstikov GA. Complexes of Polysaccharides and Glycyrrhizic Acid with Drug Molecules - Mechanochemical Synthesis and Pharmacological Activity," in The Complex World of Polysaccharides, INTECH, DN. Karunaratne, Ed., vol. 30, 2012.

[32] J.-Y. Zhou and S.-W. Zhou, "Protective effect of berberine on antioxidant enzymes and positive transcription elongation factor b expression in diabetic rat liver," Fitoterapia, vol. 82, no. 2, pp. 184-189, 2011. 

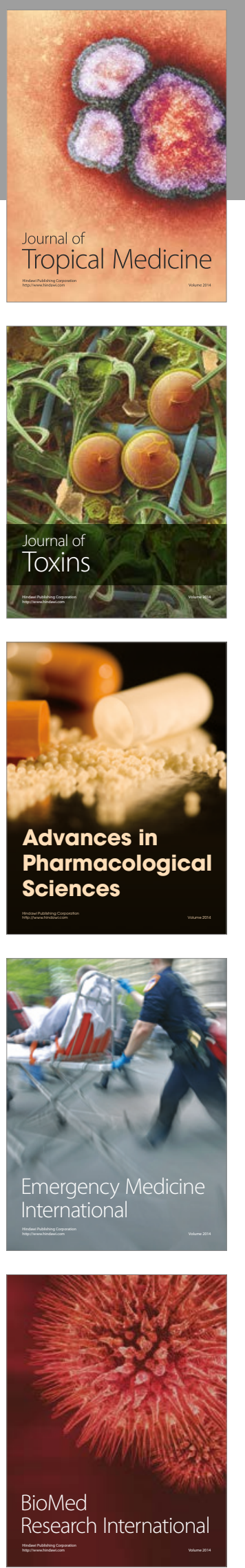
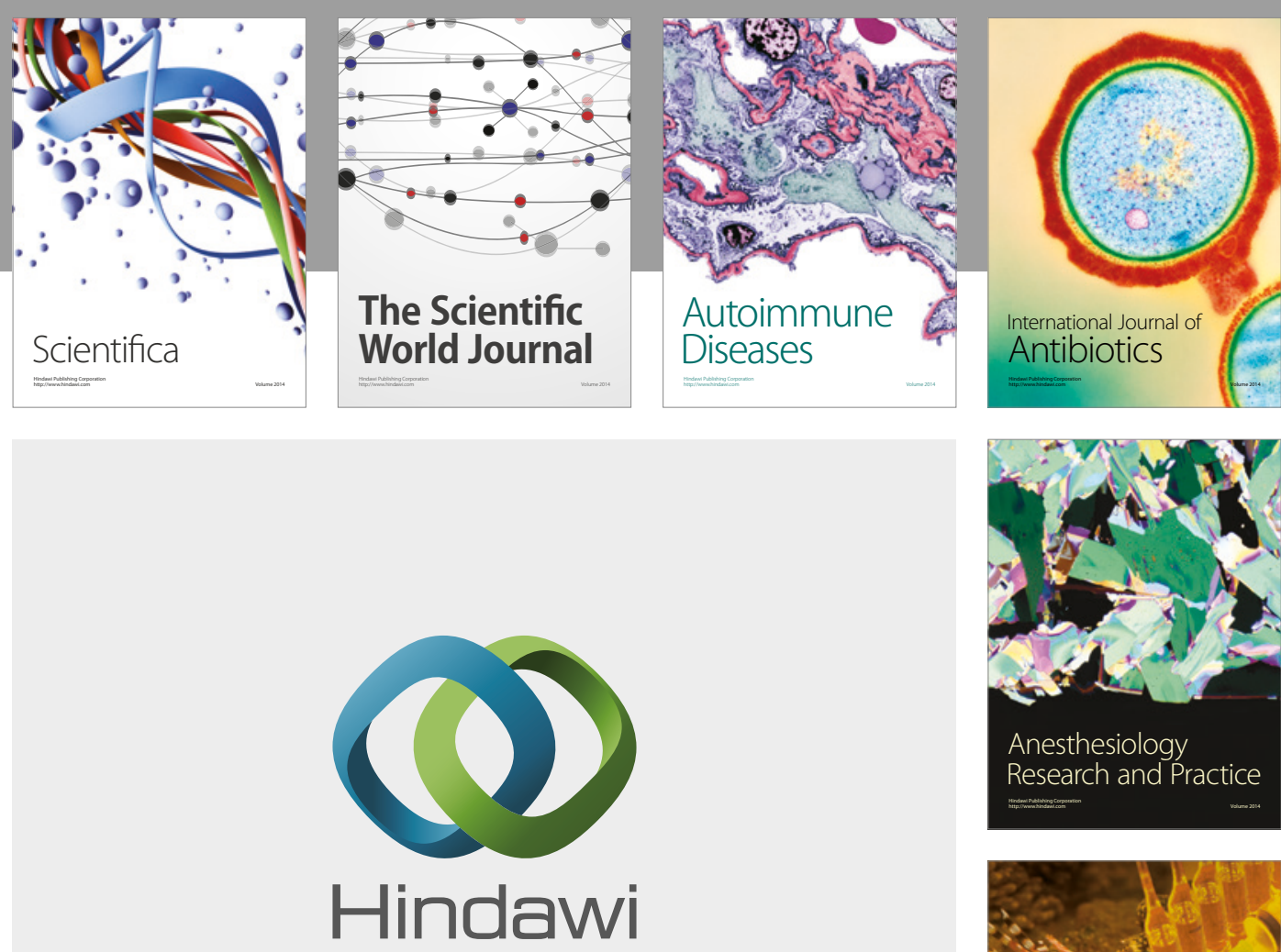

Submit your manuscripts at

https://www.hindawi.com
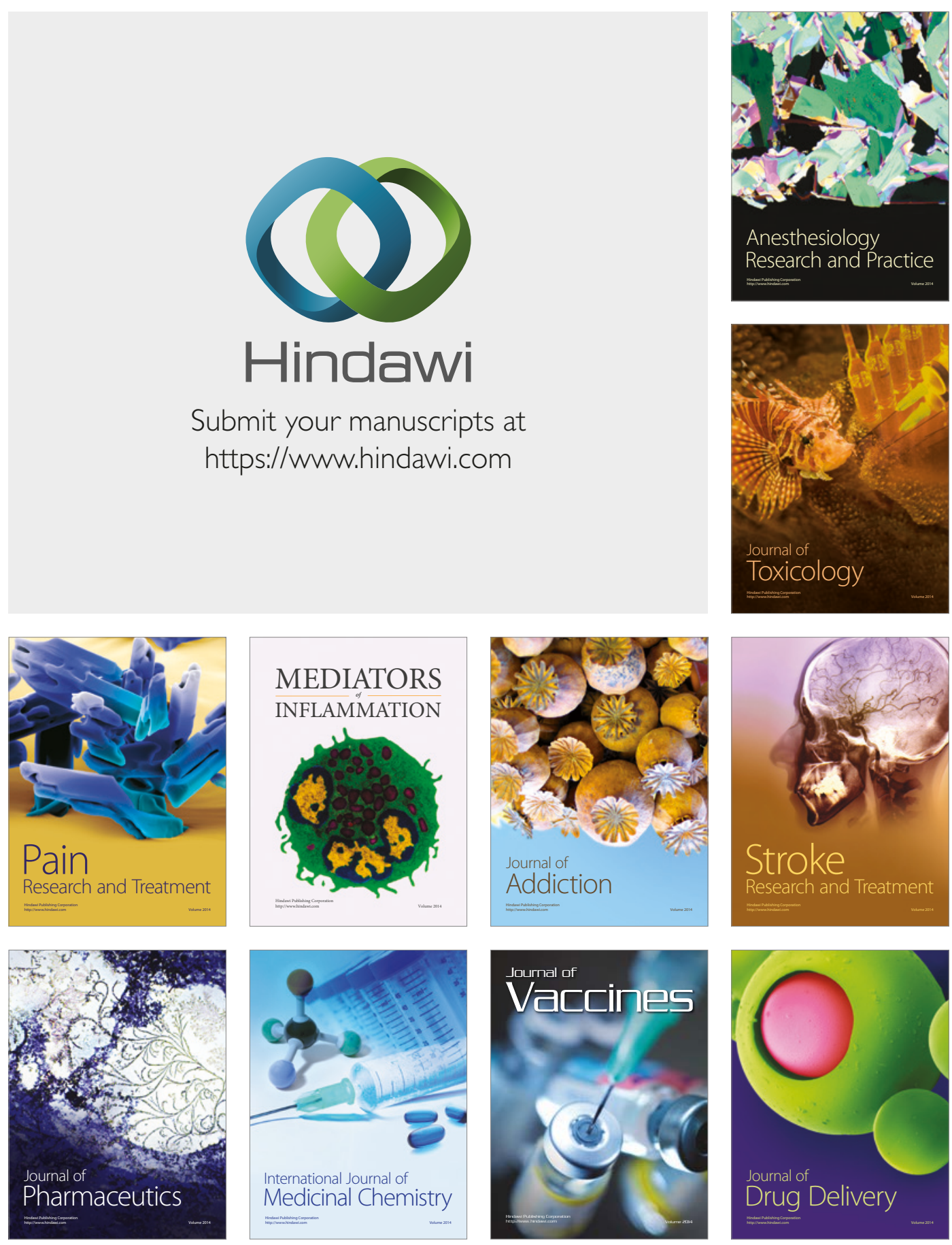